\title{
Interleukin 35 induced Th2 and Tregs bias under normal condition in mice
}

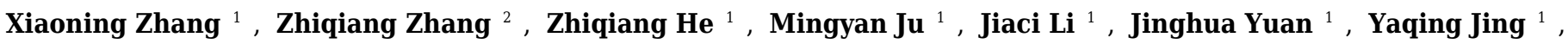

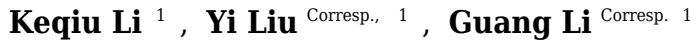 \\ 1 Department of Genetics, School of Basic Medical Sciences, Tianjin Medical University, Tianjin, China \\ 2 Department of Pathology, Tianjin Hospital of ITCWM, Nankai Hospital, Tianjin, China \\ Corresponding Authors: Yi Liu, Guang Li \\ Email address: xiaoyurly@163.com, lig@tmu.edu.cn
}

Objective: The benefits of IL-35 treatment have been verified in multiple animal models of diseases, while its influence on T cell immunity under normal condition still needs to be elucidated. The present study was designed to investigate the effects modulating IL-35 levels in vivo and in vitro on T cells response and also the effects on T cells subsets in normal mice. Methods: A plasmid pMSCV-IL-35-GFP carrying mouse linear IL-35 fragment with two subunits joint together was constructed and the heterodimer expression was confirmed. Normal mice were randomly divided into 3 groups and received an intravenous injection of PBS, pMSCV-GFP and pMSCV-IL-35-GFP respectively. After 72 hours, spleen tissues and peripheral blood were harvested for following analysis. Meanwhile, splenic $T$ cells were isolated and incubated with 10,30 , or $50 \mathrm{ng} / \mathrm{mL}$ recombinant IL-35 factor for $24 \mathrm{~h}$ with the addition of anti-CD3/CD28 in vitro. T-cell subsets were assessed by Fluorescence activated cell sorting (FACS) and related cytokines together with effector molecules were determined by real time PCR. Results: Western blotting confirmed a 52 kDa band in the cell lysate of HEK 293T transducted with pMSCV-IL-35-GFP plasmid, indicating a successful expression of IL-35. Ebi3 and IL-12A, two subunits of IL-35, could be identified 72 hours post DNA injection. IL-35 upregulation in vivo effectively inhibit CD4+ and $\mathrm{CD} 8^{+} \mathrm{T}$ cells proliferation and Th1 cytokine secretion. Effector molecules of $\mathrm{CD}^{+} \mathrm{T}$ cells were also remarkably suppressed. On the contrary, high level of IL-35 significantly induced $\mathrm{CD} 4{ }^{+} \mathrm{CD} 25^{+}$Tregs and Th2 enhancement. The in vitro study provided similar results. Conclusion: The results indicated Th1 and CD8 ${ }^{+} \mathrm{T}$ cells inhibition and Th2 and Tregs bias in the presence of IL-35 under normal state which partly at least contributed to its therapeutic potential. 
1 Interleukin 35 induced Th2 and Tregs bias under normal condition in mice

2 Xiaoning Zhang ${ }^{1}$, Zhiqiang Zhang ${ }^{2}$, Zhiqiang $\mathrm{He}^{1}$, Mingyan $\mathrm{Ju}^{1}$, Jiaci $\mathrm{Li}^{1}$, Jinghua Yuan ${ }^{1}$,

3 Yaqing Jing ${ }^{1}, \mathrm{Keqiu} \mathrm{Li}^{1}$, Yi Liu ${ }^{1,}$, Guang $\mathrm{Li}^{1,}$,

$4 \quad{ }^{1}$ Department of Genetics, School of Basic Medical Sciences, Tianjin Medical University, Tianjin, China

$5 \quad 2$ Department of Pathology, Tianjin Hospital of ITCWM, Nankai Hospital, Tianjin, China

6 * Correspondence: Yi Liu, Department of Genetics, School of Basic Medical Sciences, Tianjin Medical

7 University, 22 Qixiangtai Road, Heping District, Tianjin, 300070, People's Republic of China. E-mail:

8 xiaoyurly@163.com

\section{ABSTRACT}

Objective: The benefits of IL-35 treatment have been verified in multiple animal models of diseases, while its influence on $\mathrm{T}$ cell immunity under normal condition still needs to be elucidated. The present study was designed to investigate the effects modulating IL-35 levels in vivo and in vitro on $\mathrm{T}$ cells response and also the effects on $\mathrm{T}$ cells subsets in normal mice.

Methods: A plasmid pMSCV-IL-35-GFP carrying mouse linear IL-35 fragment with two subunits joint together was constructed and the heterodimer expression was confirmed. Normal mice were randomly divided into 3 groups and received an intravenous injection of PBS, pMSCV-GFP and pMSCV-IL-35-GFP respectively. After 72 hours, spleen tissues and peripheral blood were harvested for following analysis. Meanwhile, splenic T cells were isolated and incubated with 10,30 , or $50 \mathrm{ng} / \mathrm{mL}$ recombinant IL-35 factor for $24 \mathrm{~h}$ with the addition of antiCD3/CD28 in vitro. T-cell subsets were assessed by Fluorescence activated cell sorting (FACS) and related cytokines together with effector molecules were determined by real time PCR.

Results: Western blotting confirmed a $52 \mathrm{kDa}$ band in the cell lysate of HEK 293T transducted with pMSCV-IL-35-GFP plasmid, indicating a successful expression of IL-35. Ebi3 and IL-12A, two subunits of IL-35, could be identified 72 hours post DNA injection. IL-35 upregulation in vivo effectively inhibit $\mathrm{CD}^{+}$and $\mathrm{CD}^{+} \mathrm{T}$ cells proliferation and Th1 cytokine secretion. Effector molecules of $\mathrm{CD}^{+} \mathrm{T}$ cells were also remarkably suppressed. On the contrary, high level of IL-35 significantly induced $\mathrm{CD} 4{ }^{+} \mathrm{CD} 25^{+}$Tregs and $\mathrm{Th} 2$ enhancement. The in vitro study provided 
similar results.

Conclusion: The results indicated Th1 and $\mathrm{CD}^{+} \mathrm{T}$ cells inhibition and $\mathrm{Th} 2$ and Tregs bias in the presence of IL-35 under normal state which partly at least contributed to its therapeutic potential.

\section{Introduction}

Interleukin 35 (IL-35), a heterodimer composed of Epstein-Barr-virus-induced gene 3 (Ebi3) and interleukin-12 alpha (IL-12A), secreted by natural regulatory T cells (Tregs), is a novel cytokine of IL-12 family (Collison et al., 2007; Sawant et al., 2015). Unlike the pro-inflammatory properties of other IL-12 family members, IL-35 plays potent immunosuppressive roles partly by means of Tregs expansion, which is essential for the maintenance of immune tolerance (Collison et al., 2010). Recent studies have demonstrated the efficacy of IL-35 in inflammatory bowel disease (Wang et al., 2018), autoimmune encephalomyelitis (Choi et al., 2017; Guan et al., 2017), collagen-induced arthritis (Li et al., 2016) and acute graft-versus-host disease (Zhang et al., 2015). Our team also proved the therapeutic application of IL-35 in dextran sulfate sodium (DSS)-induced colitis (in revision). Thus, the anti-inflammatory ability makes IL-35 a promising intervention agent in inflammation, infection and other immune-related disorders.

T cells especially $\mathrm{CD} 4^{+} \mathrm{T}$ helper $(\mathrm{Th})$ cells and $\mathrm{CD} 4^{+} \mathrm{CD} 25^{+}$Tregs are critical for immuneregulation in cellular immunity and immunity homeostasis (Xiao et al., 2012; McQuillan et al., 2010). Cytokine profiles released by these cells also take part in and accelerate their action (Choi et al., 2015; Egwuagu et al., 2015). Th1 cells together with secreted IL-2 and interferon- $\gamma$ (IFN- $\gamma$ ) have been well-known to mediate inflammation and related diseases, which got significantly inhibited by IL-35 in animal disease models (Ma et al., 2014; Guo et al., 2017). Th2 and Tregs, in favor of anti-inflammation partly depending on the production of IL-10 and IL-35 respectively, received effective increase post IL-35 administration (Guo et al., 2017; Zhao et al., 2017; Bettini and Vignali, 2009). Another $\mathrm{CD}^{+} \mathrm{T}$ cells, whose function rely on cytotoxin release including

54 Granzyme B (Gzmb) and perforin 1 (Prf1) upon antigen stimulation, also received effective 
55

56

57

58

59

60

61

62

63

64

65

66

67

68

69

70

71

72

73

74

75

76

77

78

79

80

81

suppression following IL-35 use (Wong and Pamer, 2003; Milstein et al., 2011). However, most of these findings about the immune-regulation capacity of IL-35 were discovered based on disease modeling animals, the in vivo effects on normal animals have not been well described. In the present study, a plasmid carrying recombinant mouse IL-35 sequence was intravenously injected into normal mice and the short-term effect of general overexpression of IL-35 heterodimer on immunological status, particularly the differentiation of T-cell subsets, was evaluated. The results could facilitate illuminating the underlying therapeutic mechanisms of IL35 .

3

\section{Materials and methods}

\subsection{Reagents}

RPMI 1640 and fetal bovine serum (FBS) were purchased from Biological Industries (USA). Phosphate buffered saline (PBS) and erythrocyte lysis buffer were bought from Beijing Solarbio Science \& Technology Co., Ltd. (China). FuGENE transfection reagent was acquired from Promega Biosciences (USA). IL-12 p35 antibody came from Abcam (UK). TRIzol was from Thermo Fisher Scientific Inc. (USA). Phanta Super-Fidelity DNA Polymerase, HiScript II Q Select RT SuperMix for qPCR (+ gDNA wiper) and AceQ qPCR SYBR Green Master Mix were purchased from Vazyme Biotech Co., Ltd. (China). Anti-CD3 and CD28 for T cell stimulation were bought from Affinity Biosciences (USA). Anti-mouse FITC-CD4, PE-CD8 and PE-CD25 for flow cytometry were acquired from Thermo Fisher Scientific Inc. (USA). The plasmids pMSCV-GFP and pcDNA3.1-IL-35 were cryopreserved in our laboratory. Recombinant human IL-35 (rIL-35) was from PeproTech (USA).

\subsection{Animals}

Wide type male C57BL/6J mice (8- to 12-weeks old, Certificate SCK 2014-0013) were purchased from the Academy of Military Medical Sciences (Beijing, China). Mice were kept under specific pathogen free conditions according to institutional guidelines. Experimental 
82 protocols and animal care methods were subjected to approval by Animal Care and Use

83 Committee of Tianjin Medical University (TMUaMEC 2017012).

84

\subsection{Cell cultures and treatment in vitro}

86

87

88

89

90

91

92

93

94

95

96

97

98

Lymphocytes were collected from the spleen tissues of wild type C57BL/6 mice. In breif, naive T cells were cultured in RPMI 1640 without FBS, supplemented with $100 \mathrm{mg} / \mathrm{mL}$ streptomycin, $100 \mathrm{U} / \mathrm{mL}$ penicillin and $2 \mathrm{mM} \mathrm{L-glutamine}$ in a $37^{\circ} \mathrm{C}$ incubator with $5 \% \mathrm{CO} 2$ post erythrocyte lysis buffer treatment. To investigate the effect of IL-35 on T-cell subsets differentiation in vitro, recombinant human IL-35 (10, 30 or $50 \mathrm{ng} / \mathrm{mL})$ was added to the cell culture medium appended with anti-CD3 $(3 \mu \mathrm{g} / \mathrm{mL}) / \mathrm{CD} 28(2 \mu \mathrm{g} / \mathrm{mL})$. Untreated cells served as a control. After $48 \mathrm{~h}$, cells were harvested for real time PCR detection.

\subsection{Construction of the plasmid carrying IL-35 gene}

The mouse IL-35 sequence $(1.4 \mathrm{~kb})$ covering Ebi3 linked with $I l 12 a$ was a kind gift from Prof. Jiyu Ju (Weifang Medical University, Shandong). The full IL-35 coding gene was amplified by PCR and subcloned into a vector (pMSCV-IRES-GFP) by EcoRI/XhoI double digestion to construct IL-35 expression plasmid pMSCV-IL-35-GFP. The fragment was confirmed by DNA sequencing. SDS-PAGE and western blot for IL-12 p35 detection in HEK293T cells were used to confirm the recombinant IL-35 expression $48 \mathrm{~h}$ post transfected with the plasmid.

\subsection{Administration of pMSCV-IL-35-GFP}

Mice were randomly divided into 3 groups with similar mean body weight: PBS group, pMSCV group and pMSCV-IL-35 group (5 mice per group). In briefly, mice received an intravenous injection of $300 \mu \mathrm{L}$ PBS containing $50 \mu \mathrm{g}$ pMSCV-GFP, $50 \mu \mathrm{g}$ pMSCV-IL-35-GFP or nothing. After 72 hours, the spleen tissues were harvested and the tissue homogenates were lysed by erythrocyte lysis buffer to remove the red blood cells. The splenic T-cell products served for real time PCR and flow cytometry assay. 


\subsection{Real time PCR}

111 Total RNA of the spleen tissues and splenic T cells was extracted using Trizol reagent and $2 \mu \mathrm{g}$

112 RNA was reverse-transcribed using HiScript II Q Select RT SuperMix for qPCR (Vazyme 113 Biotech, China) according to the manufacturers' instructions. To evaluate the expression of 114 involved genes, quantitative real time PCR detection was undertaken with AceQ qPCR SYBR 115 Green Master Mix. All the specific primers were synthesized from Sangon Biotech Co., Ltd. 116 (Shanghai, China), and the sequences of each primer were listed in Table 1. Each sample was 117 executed and analyzed in triplicate. GAPDH was used as the endogenous control.

\subsection{Fluorescence activated cell sorting (FACS) analysis}

FACS examination, proceeded as described previously (Sun et al., 2015; Chen et al., 2016), was used to determine the subpopulations of $\mathrm{CD}^{+}, \mathrm{CD}^{+} \mathrm{T}$ cells and $\mathrm{CD} 4^{+} \mathrm{CD} 25^{+}$Treg cells in cultured T cells and splenic T cells post IL-35 treatment. In simple terms, cell suspensions under analysis were incubated with anti-mouse fluorescein isothiocyanate (FITC)-CD4, -fluorescein phycoerythrin (PE)-CD8 and -CD25 antibodies respectively at $4^{\circ} \mathrm{C}$ for $30 \mathrm{~min}$. The stainedpositive cells were assayed using flow cytometer (BD FACS Calibur, USA). FlowJo 7.6.1 software was utilized for followed data analysis.

\subsection{Statistical Analysis}

Data was presented as the mean \pm standard error of the mean (Mean \pm SEM). And statistical ANOVA was used in comparison among groups and a post-hoc contrasts by Student-NewmanKeuls test was applied to confirm the significance. Two-tailed P values $<0.05$ were considered statistically significant.

\section{Results}


136

137

138

139

140

141

142

143

144

145

146

147

148

149

150

151

152

153

154

155

156

157

158

159

160

161

162

\subsection{The constructed plasmid gained successful gene expression of IL-35}

As shown in Figure 1 A, a recombinant plasmid carrying IL-35 gene composted of Ebi3 and IL $12 a$ fragments was constructed. The expression of IL-35 was further confirmed by HEK293T transient transfection assay. SDS-PAGE and western blot for IL-12 p35 were conducted and the results both showed strong bands at about $52 \mathrm{KDa}$ in DNA transfected cell lysates (Figure $1 \mathrm{~B}$ ), indicating the successful expression of IL-35.

\subsection{IL-35 inhibited $\mathrm{CD}^{+} \mathrm{T}$ cells and promoted $\mathrm{CD}^{+} \mathrm{CD}^{+} 5^{+}$Tregs generation in vitro}

It has been reported that IL-35 could act on phenotype differentiation of $\mathrm{CD}^{+}{ }^{+} \mathrm{T}$ cells (Guan et al., 2017; Huang et al., 2017). In the present study, splenic T cells were isolated and directly treated with 10,30 , or $50 \mathrm{ng} / \mathrm{mL}$ recombinant IL-35 factor for $24 \mathrm{~h}$ in the presence of antiCD3/CD28 ex vivo. The cells were then collected to assess the proportion of T-cell subsets by FACS. The results showed that $\mathrm{CD}^{+} \mathrm{T}$ cell population substantially raised upon activation, which got effectively reduced post rIL-35 supplement (Figure $2 \mathrm{~A}$ ). Also, the $\mathrm{CD} 4^{+} \mathrm{CD} 25^{+}$Tregs proportion decreased when exposed to anti-CD3/CD28, but increased after recombinant IL-35 treatment (Figure 2 B). In addition, the rIL-35 affect showed some dose-dependence, indicating that IL-35 could inhibit CD4+ T cells proliferation and generate CD4 ${ }^{+} \mathrm{CD} 25^{+}$Tregs.

\subsection{IL-35 facilitated Th2 and Tregs function in vitro.}

Cytokines produced by Th and Treg cells also take part in and play important roles in immuneregulation (Ma et al., 2014). The total RNA of cultured T cells mentioned above were extracted and the cytokine expression involved Th1 (Il2). Th2 (Il10) and Tregs (Foxp3) were examined by real time PCR. Data indicated that rIL-35 administration significantly reduced II2 level and sharply boosted I110 and Foxp3 expression in a dose-dependent manner (Figure 3), which suggested that IL-35 treatment facilitated Th2 and Tregs function.

\subsection{IL-35 overexpression inhibited $\mathrm{CD4}^{+}$and $\mathrm{CD8}^{+} \mathrm{T}$ cells but enhanced $\mathrm{CD4}^{+} \mathrm{CD25}^{+} \mathrm{Tregs}^{-}$}


163

164

165

166

167

168

169

170

171

172

173

174

175

176

177

178

179

180

181

182

183

184

185

186

187

188

189

\section{in vivo}

To further investigate the effect of IL-35 on the $\mathrm{T}$ cells differentiation in vivo, wild type C57BL/6J mice received an intravenous injection of the plasmid pMSCV-IL-35-GFP. After 72 hours, peripheral blood was sampled to test the expression of Ebi3 and $I l 12 a$ fragments by real time PCR. Spleen tissues were used to estimate the proportion of T-cell subsets by FACS. The results demonstrated both significant increases in Ebi3 and $I l 12 a$ levels in mice received pMSCV-IL-35-GFP injection, suggesting the expression of exogenous gene sequence and upregulation of IL-35 (Figure 4). The same as Figure 2 described, FACS analysis showed obviously lowered percentage of total $\mathrm{CD}^{+}$and $\mathrm{CD}^{+} \mathrm{T}$ cells (Figure $5 \mathrm{~A}-\mathrm{D}$ ) but considerably amplified $\mathrm{CD}^{+} \mathrm{CD}^{+} 5^{+}$Tregs (Figure 5E, F) compared to PBS group. Consistent with previous reports, Treg cells proliferated in response to IL-35 upregulation (Castellani et al., 2010).

\subsection{Upregulation of IL-35 increased Th2 cytokine production in vivo}

In this study, we also measured the mRNA levels of cytokines and genes related to Th1, Th2, $\mathrm{CD}^{+}$and Tregs using real time PCR. The results showed that Th1 cytokines IL-2 and IFN- $\gamma$ (Figure 6 A, B) as well as Gzmb and Prfl in $\mathrm{CD}^{+} \mathrm{T}$ cells (Figure $6 \mathrm{D}, \mathrm{E}$ ) obviously increased in mice received pMSCV plasmid. While, the generous expression of IL-35 effectively inhibited the aforementioned effector molecules. Besides, the splenic levels of $1 l 10$ (Figure $6 \mathrm{C}$ ) and Foxp3 (Figure $6 \mathrm{~F}$ ) of pMSCV-IL-35 group both got remarkably elevated. Therefore, IL-35 could effectively suppress Th1 and $\mathrm{CD}^{+} \mathrm{T}$ cell function, but strengthen Th2 and Tregs.

\section{Discussion}

In the present investigation, a plasmid loading IL-35 linear gene containing EBI3 joint with I112a was intravenously injected into normal mice to determine the effect of IL-35 heterodimer overexpression on $\mathrm{T}$ cell immunity and $\mathrm{T}$ cell differentiation under normal condition. Ebi3 and IL-12A, two subunits of IL-35, could be identified 72 hours post DNA injection. IL-35 upregulation in vivo effectively inhibited $\mathrm{CD}^{+}$and $\mathrm{CD}^{+} \mathrm{T}$ cells proliferation and $\mathrm{Th} 1$ cytokine 
190

191

192

193

194

195

196

197

198

199

200

201

202

203

204

205

206

207

208

209

210

211

212

214

215

216

213 Some works proposed that the Tregs and Th2 augment partly at least contributed to the

secretion. On the contrary, elevated level of IL-35 significantly induce CD4 ${ }^{+} \mathrm{CD} 25^{+}$Tregs and Th2 enhancement. The in vitro study provided similar results, suggesting the immunosuppressive action of IL-35 on T-cell subsets evolution. All of the data indicated the Th1 and CD8 ${ }^{+} \mathrm{T}$ cells suppression but Th2 and Tregs bias in the presence of IL-35.

As we all know, $\mathrm{T}$ cells, roughly divided into $\mathrm{CD}^{+}{ }^{+} \mathrm{Th}$ cells, $\mathrm{CD} 8^{+}$cytotoxic $\mathrm{T}$ cells (CTLs) and Tregs according to the functional distinction, play a central role in cell-mediated immunity (Milstein et al., 2011; Overgaard et al., 2015). Cytokine profiles secreted by these cells further augment their immunoregulatory capacity and act on other subsets (Biron and Tarrio, 2015). In brief, IL-2 and IFN- $\gamma$ produced by Th1 cells can enhance the cytotoxic effect of $\mathrm{CD}^{+} \mathrm{T}$ cells, which can be well inhibited by IL-10 released from Th2 cells and/or IL-35 synthesized by Tregs (Guo et al., 2017; Biron and Tarrio, 2015). The ability of Th1 and Th2 cytokines has been clearly stated, and recent studies focused on the newly discovered immunosuppressive factor IL35 .

IL-35, a member of the IL-12 family, is a heterodimeric cytokine composed of the EBI3 and IL-12A assembled in innate Tregs (Collison et al., 2007; Niedbala et al., 2007). The antiinflammatory capacity of IL-35 has been investigated in several inflammatory disorder models, such as infection diseases, organ transplantation rejection and autoimmunity diseases (Guan et al., 2017; Sun et al., 2015; Liu et al., 2014; Li et al., 2012). Evidence from these animal models showed that IL-35 administration suppressed inflammation related $\mathrm{T}$ cell differentiation. However, the existing results are mainly obtained from disease models, it still remains to be determined whether IL-35 could affect the T subsets evolution without antigen stimulation. The present study primarily aimed at expounding the impact of IL-35 on T cell differentiation and function in normal healthy mice. therapeutic potential of IL-35 (Zhao et al., 2017). To confirm this finding, activated T cells were treated with recombinant IL-35 factor for $24 \mathrm{~h}$ ex vivo. The data indicated that IL-35 remarkably suppressed $\mathrm{CD}^{+} \mathrm{T}$ cell proliferation (Figure $2 \mathrm{~A}$ ) and IL-2 release (Figure $3 \mathrm{~A}$ ). Instead, 
$217 \mathrm{CD}^{+} \mathrm{CD} 25^{+}$Tregs (Figure $2 \mathrm{~B}$ ) and IL-10 secretion (Figure 3 B) by Th2 cells got effective 218 increase. Foxp3 also showed a distinct growth under treatment with 50ng/mL IL-35 factor 219 (Figure $3 \mathrm{C}$ ). The in vivo effect of IL-35 on T-cell subsets development was then investigated 220 after a plasmid carrying IL-35 fragment (Figure 1) administration in normal mice. The much 221 higher expression of Ebi3 and IL-12p35 in mice received pMSCV-IL-35 plasmid could be 222 observed three days later (Figure 4). Similarly, both splenic $\mathrm{CD} 4^{+}$and $\mathrm{CD} 8^{+} \mathrm{T}$ cells performed 223 significantly restrained (Figure $5 \mathrm{~A}-\mathrm{D}$ ), but $\mathrm{CD} 4{ }^{+} \mathrm{CD} 25^{+}$Tregs got well amplification (Figure $5 \mathrm{E}$, 224 F). Related cytokine and effector molecule analysis further confirmed lower IL-2 and IFN- $\gamma$ level 225 (Figure 6 A, B) together with largely reduced Gzmb and Prf1 production (Figure 6 D, E) upon 226 IL-35 expression. In contrast, both IL-10 (Figure 6 C) and Foxp3 (Figure 6 F) increased

227 228 229 230 231 232 233 234 235 236

\section{Conclusions}

238

239

240

241

242 significantly. Thus, IL-35 overexpression indeed affect $\mathrm{T}$ cell differentiation and induced an obvious Th2 and Tregs bias.

The suppressive capacity of IL-35 has been tested in many disease models, still the influence of general IL-35 administration on normal body needs to be clarified. Data listed here based on normal healthy mice could partly help to understand how IL-35 work and the potential therapeutic mechanisms in disease cases. And it's important to note that, the present study was conducted in normal animals and the outcome might have some difference from those in pathologic condition. The cure mechanisms of IL-35 treatment in various disease models still need further researches.

The present study explored the effect of recombinant heterodimer IL-35 on T-cell subset developing in normal mice for the first time. The results indicated that IL-35 upregulation could effectively induce Th2 and Tregs bias and inhibit Th1 and $\mathrm{CD}^{+} \mathrm{T}$ cell function in healthy animals. While, further studies concerning long-term effect and related mechanisms are still required to elucidate in IL-35 application. 
244

245

246

247

248

249

250

251

252

253

254

255

256

257

258

259

260

261

262

263

264

265

266

267

268

269

270

271

272

273

274

275

276

277

278

279

280

281

282

283

284

\section{References}

Collison L. W., Workman C. J., Kuo T. T., Boyd K., Wang Y., Vignali K. M., Cross R., Sehy D., Blumberg R. S. and Vignali D. A. 2007. The inhibitory cytokine IL-35 contributes to regulatory T-cell function. Nature 450(7169): 566-9 DOI 10.1038/nature06306.

Sawant D. V., Hamilton K. and Vignali D. A. 2015. Interleukin-35: Expanding Its Job Profile. J Interferon Cytokine Res 35(7): 499-512 DOI 10.1089/jir.2015.0015.

Collison L. W., Chaturvedi V., Henderson A. L., Giacomin P. R., Guy C., Bankoti J., Finkelstein D., Forbes K., Workman C. J., Brown S. A., Rehg J. E., Jones M. L., Ni H. T., Artis D., Turk M. J. and Vignali D. A. 2010. IL-35-mediated induction of a potent regulatory T cell population. Nat Immunol 11(12): 1093-101 DOI 10.1038/ni.1952.

Wang Y., Mao Y., Zhang J., Shi G., Cheng L., Lin Y., Li Y., Zhang X., Zhang Y., Chen X., Deng J., Su X., Dai L., Yang Y., Zhang S., Yu D., Wei Y. and Deng H. 2018. IL-35 recombinant protein reverses inflammatory bowel disease and psoriasis through regulation of inflammatory cytokines and immune cells. J Cell Mol Med 22(2): 1014-1025 DOI 10.1111/jcmm.13428.

Choi J. K., Dambuza I. M., He C., Yu C. R., Uche A. N., Mattapallil M. J., Caspi R. R. and Egwuagu C. E. 2017. IL-12p35 Inhibits Neuroinflammation and Ameliorates Autoimmune Encephalomyelitis. Front Immunol 8: 1258 DOI 10.3389/fimmu.2017.01258.

Guan S. Y., Leng R. X., Khan M. I., Qureshi H., Li X. P., Ye D. Q. and Pan H. F. 2017. Interleukin-35: a Potential Therapeutic Agent for Autoimmune Diseases. Inflammation 40(1): 303-310 DOI 10.1007/s10753016-0453-9.

Li Y., Wu S., Li Y., Jiang S., Lin T., Xia L., Shen H. and Lu J. 2016. Interleukin-35 (IL-35) inhibits proliferation and promotes apoptosis of fibroblast-like synoviocytes isolated from mice with collagen-induced arthritis. Mol Biol Rep 43(9): 947-56 DOI 10.1007/s11033-016-4034-7.

Zhang X. H., Zhou Y., Zhang J. M., Zhou S. Y., Wang M., Feng R., Feng F. E., Wang Q. M., Zhu X. L., Zhao X. S., Lv M., Kong Y., Chang Y. J. and Huang X. J. 2015. IL-35 inhibits acute graft-versus-host disease in a mouse model. Int Immunopharmacol 29(2): 383-392 DOI 10.1016/j.intimp.2015.10.025.

Xiao J., Zhu F., Liu X. and Xiong J. 2012. Th1/Th2/Th17/Treg expression in cultured PBMCs with antiphospholipid antibodies. Mol Med Rep 6(5): 1035-9 DOI 10.3892/mmr.2012.1055.

McQuillan K., Lynch M. A. and Mills K. H. 2010. Activation of mixed glia by Abeta-specific Th1 and Th17 cells and its regulation by Th2 cells. Brain Behav Immun 24(4): 598-607 DOI 10.1016/j.bbi.2010.01.003.

Choi J., Leung P. S., Bowlus C. and Gershwin M. E. 2015. IL-35 and Autoimmunity: a Comprehensive Perspective. Clin Rev Allergy Immunol 49(3): 327-32 DOI 10.1007/s12016-015-8468-9.

Egwuagu C. E., Yu C. R., Sun L. and Wang R. 2015. Interleukin 35: Critical regulator of immunity and lymphocyte-mediated diseases. Cytokine Growth Factor Rev 26(5): 587-93 DOI 10.1016/j.cytogfr.2015.07.013.

Ma L., Liang Y., Fang M., Guan Y., Si Y., Jiang F. and Wang F. 2014. The cytokines (IFN-gamma, IL-2, IL-4, IL-10, IL-17) and Treg cytokine (TGF-beta1) levels in adults with immune thrombocytopenia. Pharmazie 69(9): 694-7 DOI

Guo H., Zhao N., Gao H. and He X. 2017. Mesenchymal Stem Cells Overexpressing Interleukin-35 Propagate Immunosuppressive Effects in Mice. Scand J Immunol 86(5): 389-395 DOI 10.1111/sji.12613.

Zhao N., Li H., Yan Y., Jiang R. and He X. 2017. Mesenchymal stem cells overexpressing IL-35 effectively 
inhibit CD4(+) T cell function. Cell Immunol 312: 61-66 DOI 10.1016/j.cellimm.2016.12.001.

Bettini M. and Vignali D. A. 2009. Regulatory T cells and inhibitory cytokines in autoimmunity. Curr Opin Immunol 21(6): 612-8 DOI 10.1016/j.coi.2009.09.011.

Wong P. and Pamer E. G. 2003. CD8 T cell responses to infectious pathogens. Annu Rev Immunol 21: 29-70 DOI 10.1146/annurev.immunol.21.120601.141114.

Milstein O., Hagin D., Lask A., Reich-Zeliger S., Shezen E., Ophir E., Eidelstein Y., Afik R., Antebi Y. E., Dustin M. L. and Reisner Y. 2011. CTLs respond with activation and granule secretion when serving as targets for T-cell recognition. Blood 117(3): 1042-52 DOI 10.1182/blood-2010-05-283770.

Sun T., Zhang D., Yang Y., Zhang X., Lv C., Fu R., Lv M., Liu W., Chen Y., Liu W., Huang Y., Xue F., Liu X., Zhang L., Li H. and Yang R. 2015. Interleukin 35 may contribute to the loss of immunological selftolerance in patients with primary immune thrombocytopenia. Br J Haematol 169(2): 278-85 DOI 10.1111/bjh.13292.

Chen D., Li Y., Wang X., Li K., Jing Y., He J., Qiang Z., Tong J., Sun K., Ding W., Kang Y. and Li G. 2016. Generation of regulatory dendritic cells after treatment with paeoniflorin. Immunol Res 64(4): 988-1000 DOI $10.1007 / \mathrm{s} 12026-015-8773-7$.

Huang A., Cheng L., He M., Nie J., Wang J. and Jiang K. 2017. Interleukin-35 on B cell and T cell induction and regulation. J Inflamm (Lond) 14: 16 DOI 10.1186/s12950-017-0164-5.

Castellani M. L., Anogeianaki A., Felaco P., Toniato E., De Lutiis M. A., Shaik B., Fulcheri M., Vecchiet J., Tete S., Salini V., Theoharides T. C., Caraffa A., Antinolfi P., Frydas I., Conti P., Cuccurullo C., Ciampoli C., Cerulli G. and Kempuraj D. 2010. IL-35, an anti-inflammatory cytokine which expands CD4+CD25+ Treg Cells. J Biol Regul Homeost Agents 24(2): 131-5 DOI

Overgaard N. H., Jung J. W., Steptoe R. J. and Wells J. W. 2015. CD4+/CD8+ double-positive T cells: more than just a developmental stage? J Leukoc Biol 97(1): 31-8 DOI 10.1189/jlb.1RU0814-382.

Biron C. A. and Tarrio M. L. 2015. Immunoregulatory cytokine networks: 60 years of learning from murine cytomegalovirus. Med Microbiol Immunol 204(3): 345-54 DOI 10.1007/s00430-015-0412-3.

Niedbala W., Wei X. Q., Cai B., Hueber A. J., Leung B. P., McInnes I. B. and Liew F. Y. 2007. IL-35 is a novel cytokine with therapeutic effects against collagen-induced arthritis through the expansion of regulatory $\mathrm{T}$ cells and suppression of Th17 cells. Eur J Immunol 37(11): 3021-9 DOI 10.1002/eji.200737810.

Liu Y., Wu Y., Wang Y., Cai Y., Hu B., Bao G., Fang H., Zhao L., Ma S., Cheng Q., Song Y., Zhu Z., Chang H., Yu X., Sun A., Zhang Y., Vignali D. A., Wu D. and Liu H. 2014. IL-35 mitigates murine acute graftversus-host disease with retention of graft-versus-leukemia effects. Leukemia: $\quad$ DOI 10.1038/leu.2014.310.

Li X., Mai J., Virtue A., Yin Y., Gong R., Sha X., Gutchigian S., Frisch A., Hodge I., Jiang X., Wang H. and Yang X. F. 2012. IL-35 is a novel responsive anti-inflammatory cytokine--a new system of categorizing antiinflammatory cytokines. PLoS One 7(3): e33628 DOI 10.1371/journal.pone.0033628. 


\section{Figure 1}

Characterization of mouse IL-35 expression.

(A) Construction schematic of pMSCV-IL-35-GFP (7949 bp) encoding the recombinant IL-35.

(B) IL-35 expression ( $52 \mathrm{kDa}$ ) was confirmed by SDS-PAGE and western blotting 48 hours post transfection into HEK 293T cells.

A

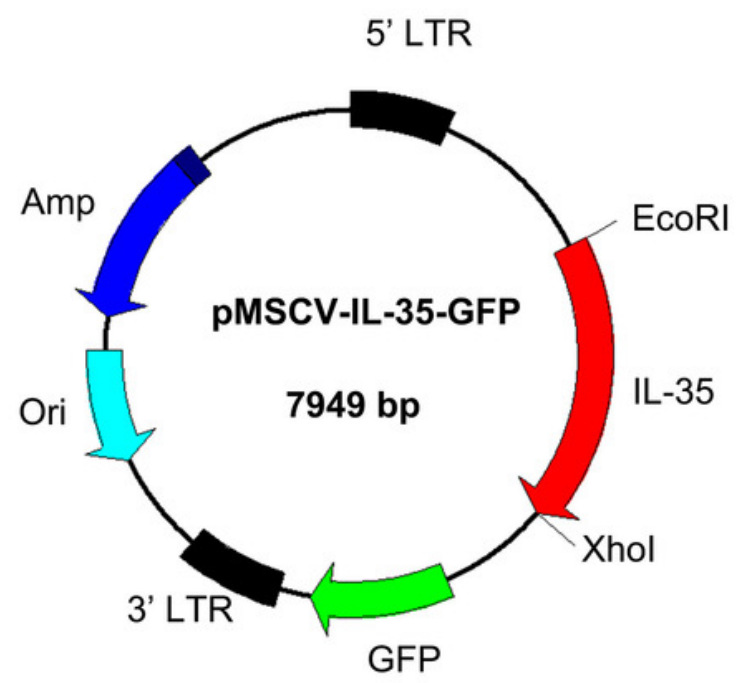

B

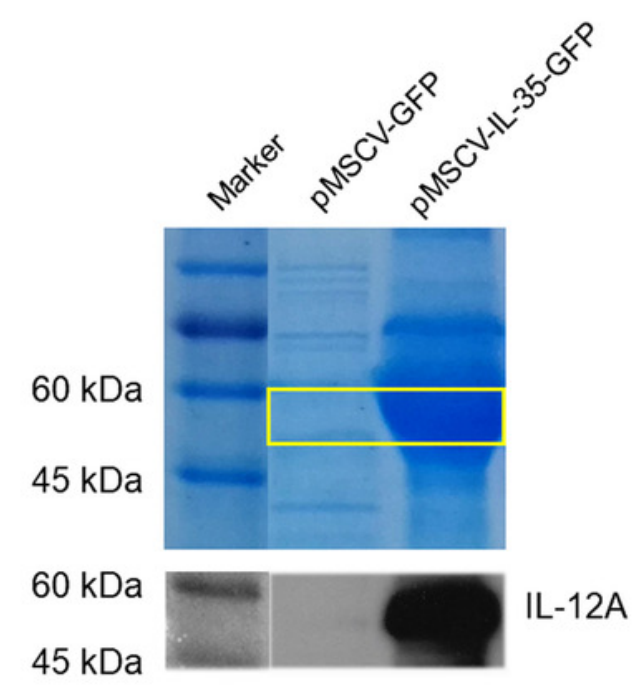


Figure 2

The phenotype of total $\mathrm{CD} 4^{+} \mathrm{T}$ cells and $\mathrm{CD} 4^{+} \mathrm{CD} 25^{+}$Tregs detected by FACS.

(A) $\mathrm{CD} 4^{+} \mathrm{T}$ cells proliferation got effective inhibition with rIL-35 addition. (B) IL-35 promoted the amplification of $\mathrm{CD} 4^{+} \mathrm{CD} 25^{+}$Tregs. Both the effect on proliferation of $\mathrm{CD} 4^{+} \mathrm{T}$ cells and $\mathrm{CD} 4^{+} \mathrm{CD} 25^{+}$Tregs appeared some dose-dependent manner.

A
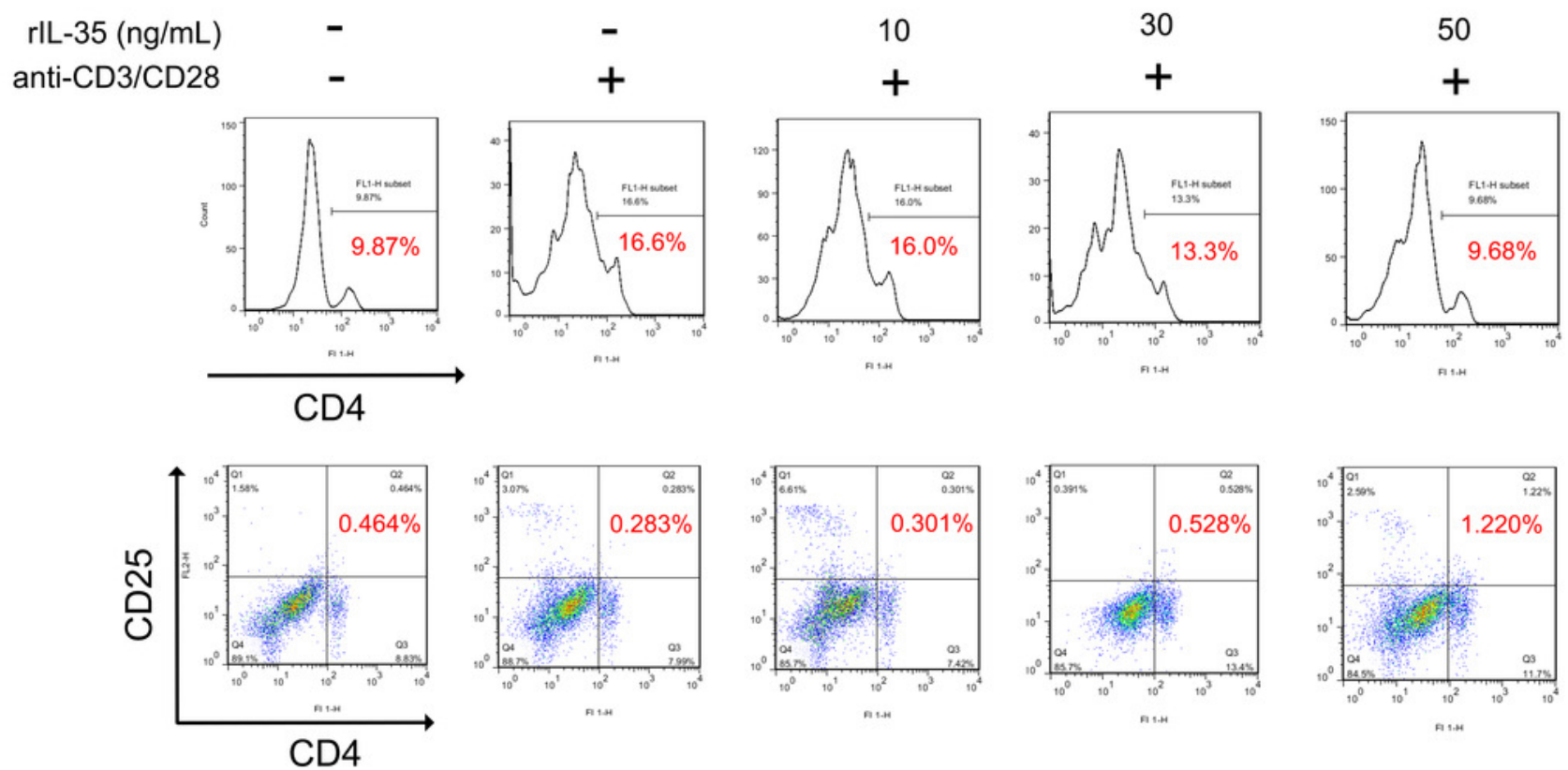
Figure 3

The expression of $112, \| 10$, and Foxp3 in splenic T cells following rlL-35 addition for $24 \mathrm{~h}$ in vitro.

Remarkably lower II2 level (A) but multiplied II10 (B) and Foxp3 (C) could be observed with rIL-35 treatment, signifying restrained Th1 but promotion of Th2 and Tregs. ${ }^{*} P<0.05$, ${ }^{* *} P<0.01,{ }^{* * *} P<0.001$ vs. control: anti-CD3/CD28 (+) rlL-35 (-).
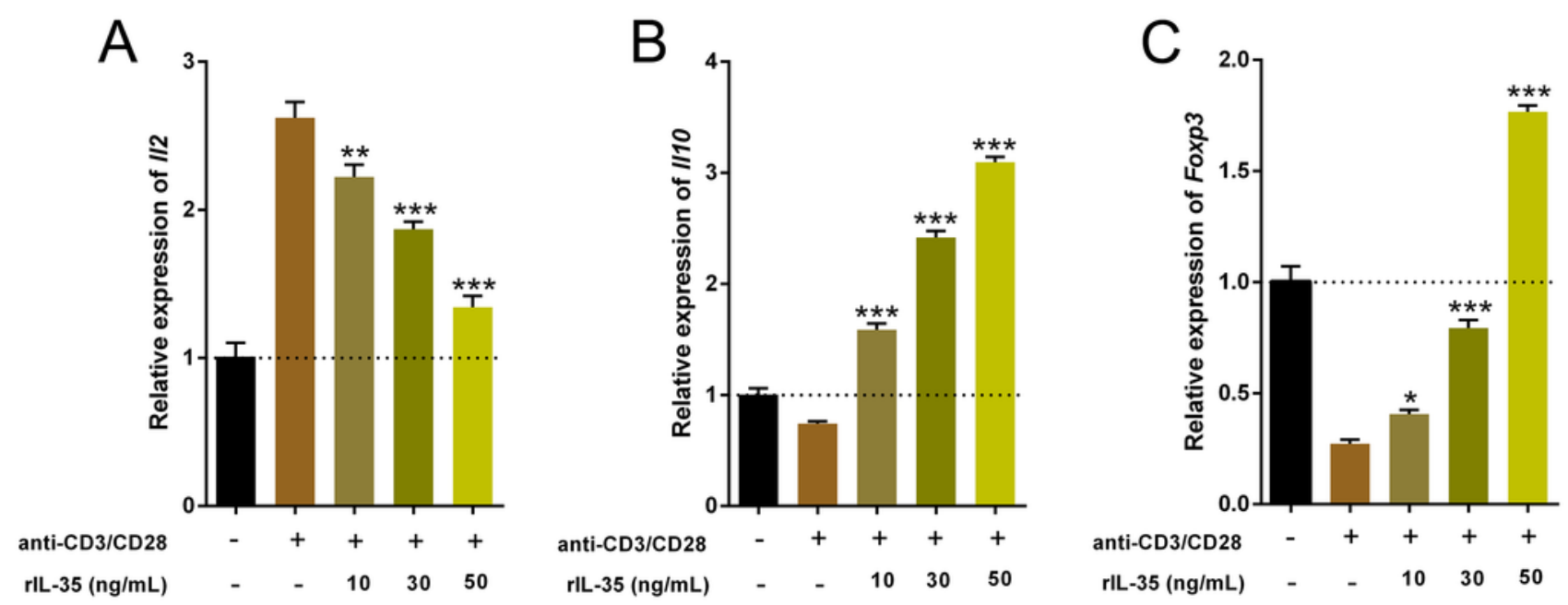


\section{Figure 4}

The transcriptional levels of Ebi3 and I/12a 72 hours post plasmid pMSCV-IL-35-GFP injection.

Both Ebi3 and II12a, the subunits of IL-35, got nearly doubled in mice exposed to IL-35carrying plasmid, indicating a successful expression of exogenous IL-35. ${ }^{* *} P<0.01,{ }^{* * *} P<0.001$ vs. PBS group, ${ }^{\# \#} P<0.001$ vs. pMSCV group. 


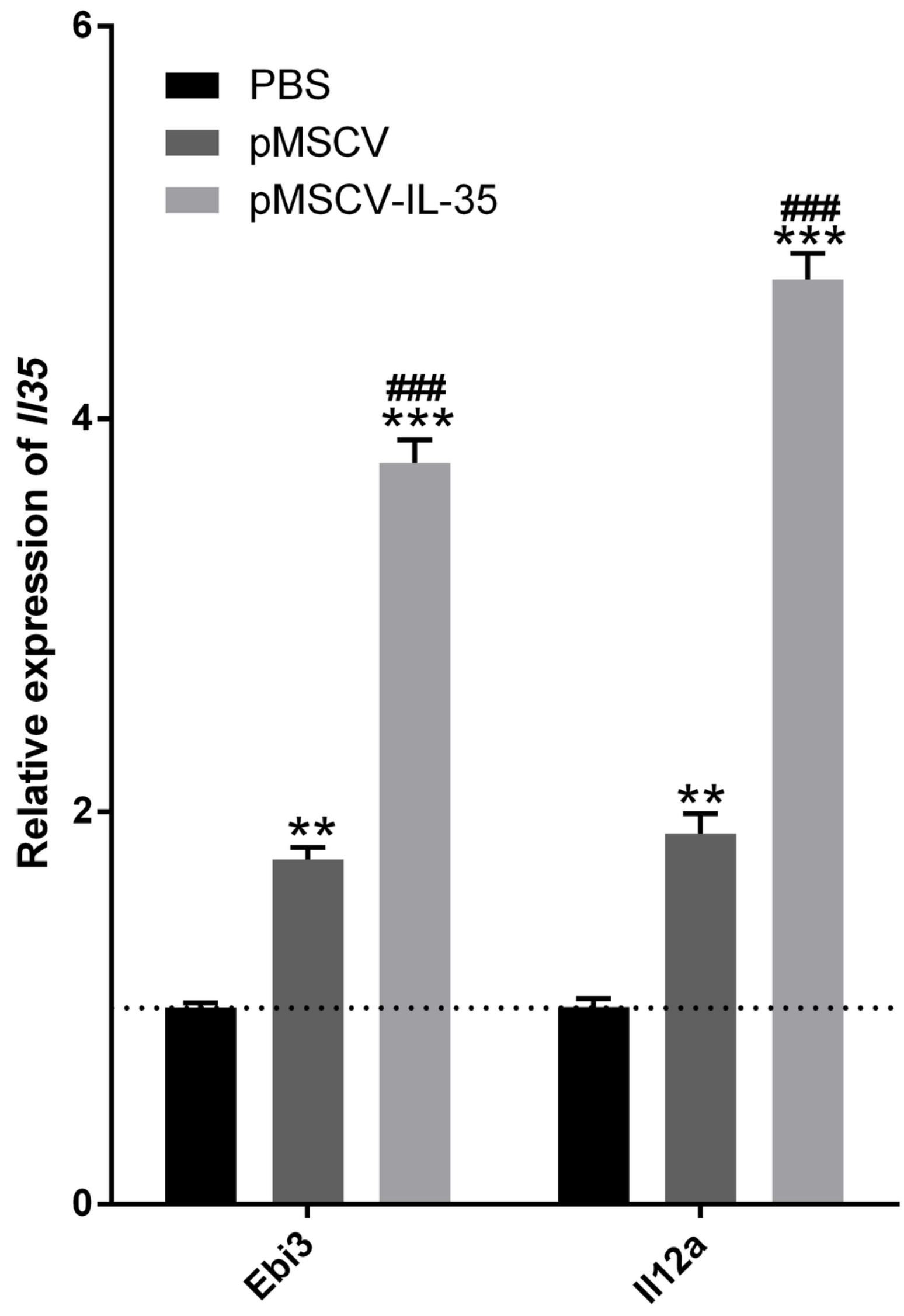


Figure 5

The $\mathrm{CD} 4^{+}, \mathrm{CD} 8^{+} \mathrm{T}$ cells and $\mathrm{CD} 4^{+} \mathrm{CD} 25^{+}$Tregs subsets.

(A), (C) and (E) exhibited FACS detection and (B), (D) and (F) showed the statistical data. IL35 overexpression in vivo clearly inhibited both $\mathrm{CD} 4^{+}$and $\mathrm{CD} 8^{+} \mathrm{T}$ proliferating but significantly enhanced $\mathrm{CD} 4{ }^{+} \mathrm{CD} 25^{+}$Tregs. ${ }^{*} P<0.05,{ }^{* * *} P<0.001$ vs. PBS group, ${ }^{*} P<0.05$,

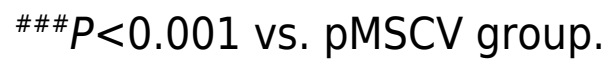

A

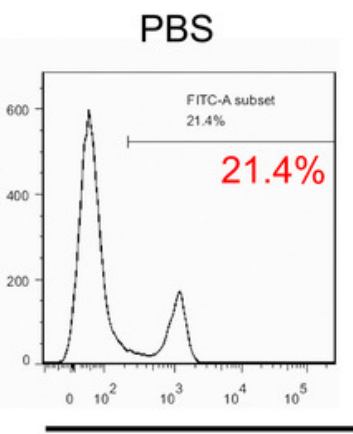

C

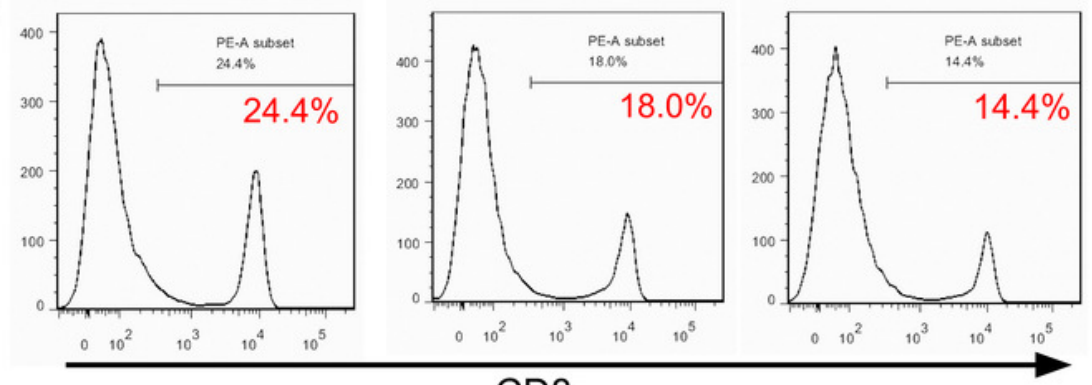

E

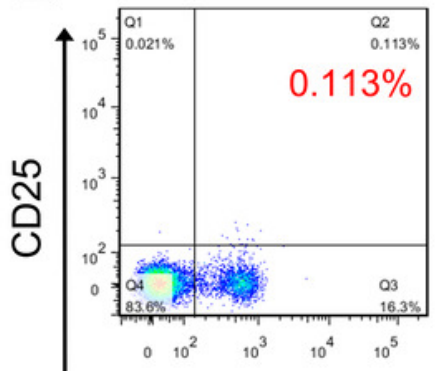

pMSCV

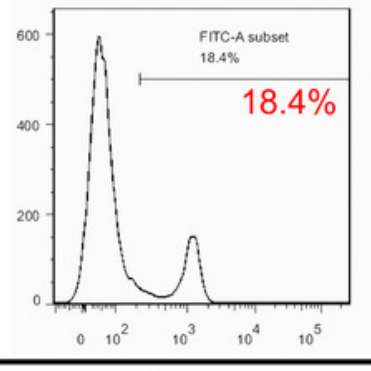

CD8

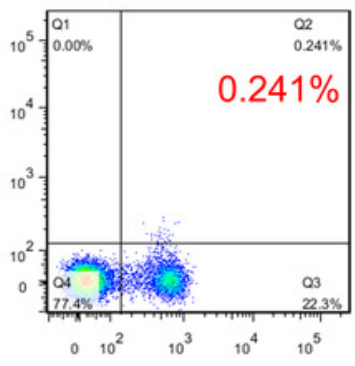

CD4
B

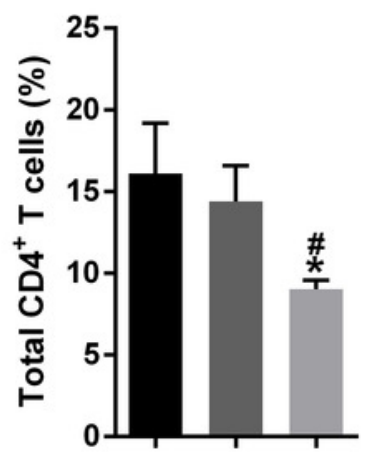

D

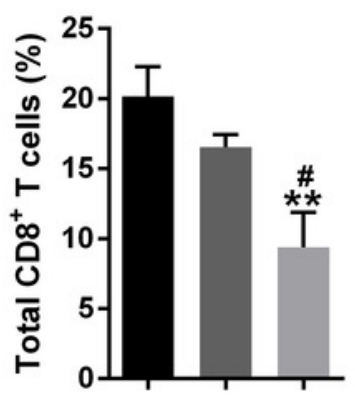

F

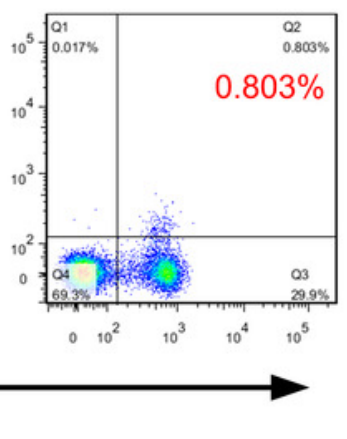

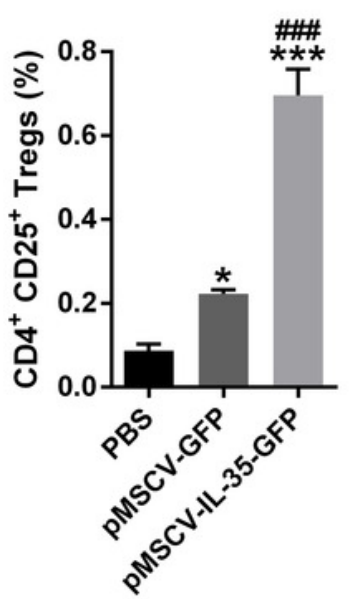


Figure 6

The mRNA level of II2, Ifng, II10, Gzmb, Prf1, and Foxp3 in vivo.

Th1 cytokines including (A) II2 and (B) Ifng together with (D) Gzmb and (E) Prf1 in CD8+ T cells all got remarkably inhibited, while II10 (C) produced by Th2 cells and Foxp3 (F) in CD4 ${ }^{+}$ CD25+ Tregs received cleared upregulation. ${ }^{*} P<0.05,{ }^{* *} P<0.01,{ }^{* * *} P<0.001$ vs. PBS group,

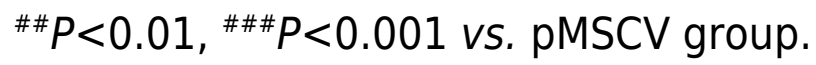

A

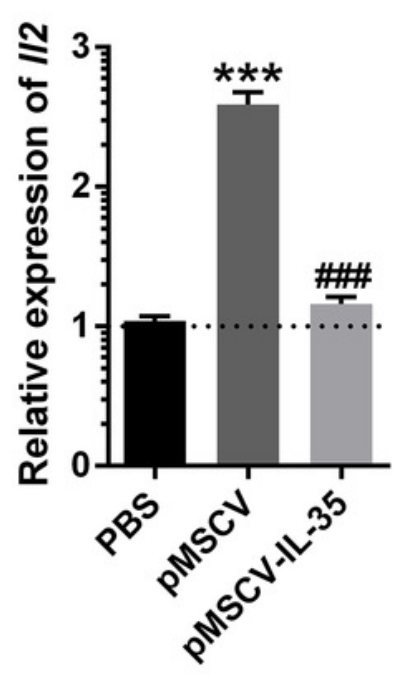

D

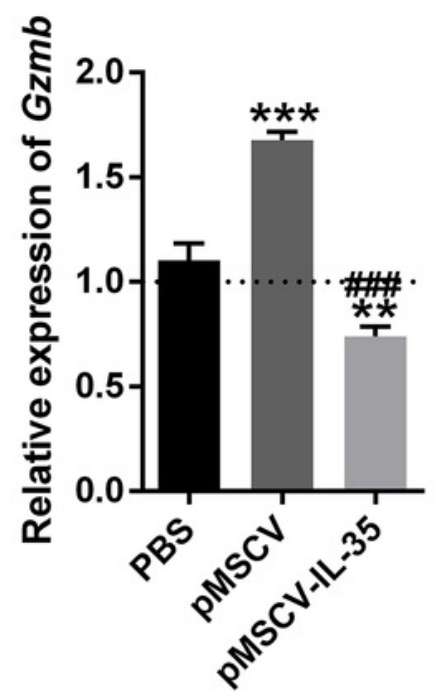

B

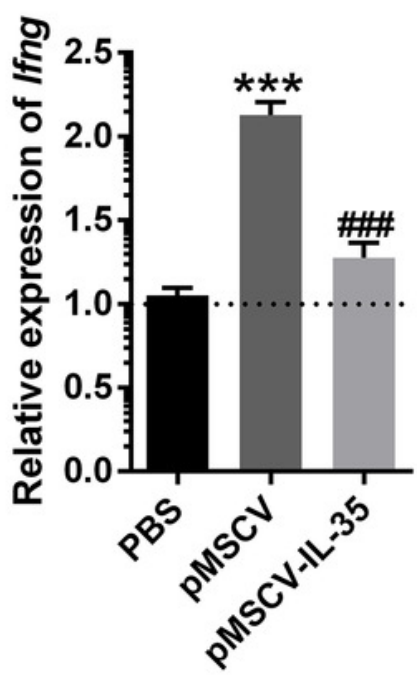

$E$

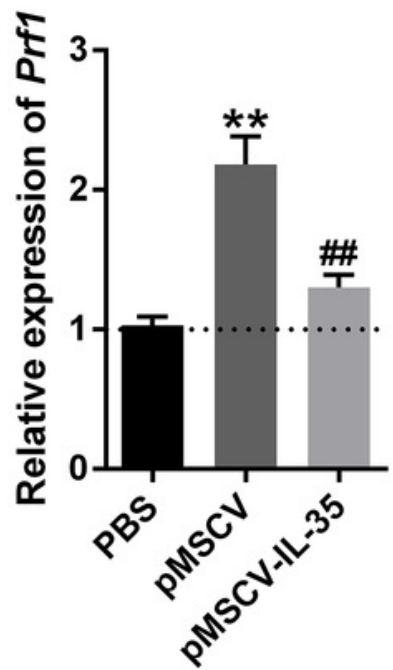

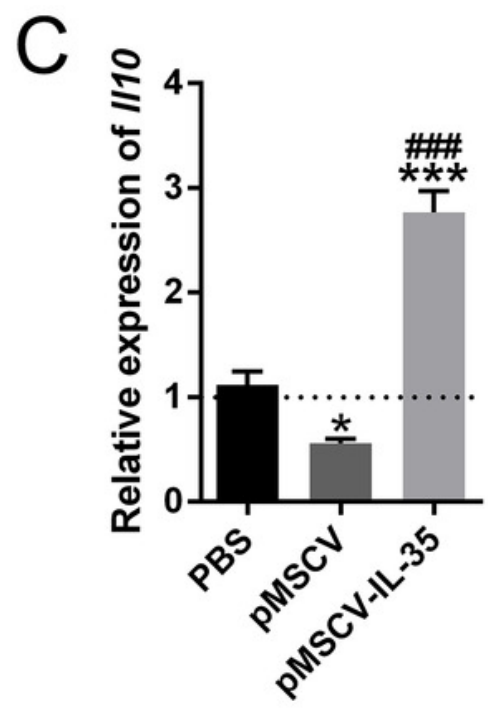

$\mathrm{F}$

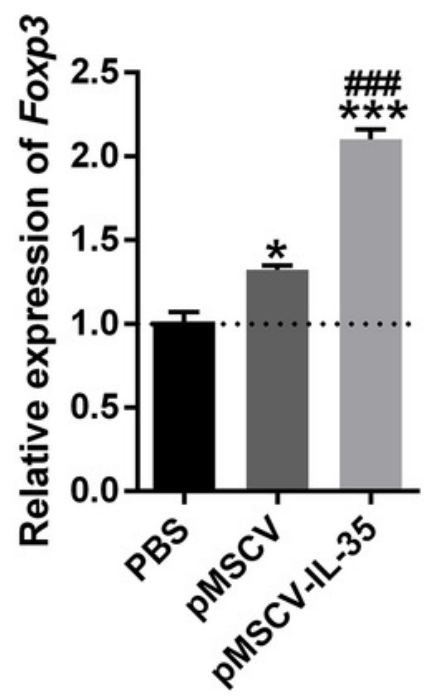


Table $\mathbf{1}$ (on next page)

Primer sequences for real time PCR detection 
Table 1 Primer sequences for real time PCR detection

\begin{tabular}{llll}
\hline Genes & Sense primers $\left(5^{\prime} \rightarrow 3^{\prime}\right)$ & Antisense primers $\left(5^{\prime} \rightarrow 3^{\prime}\right)$ & $\begin{array}{l}\text { Product } \\
\text { length }(\mathrm{bp})\end{array}$ \\
\hline Ebi3 & GTT CTC CAC GGT GCC CTA & CGG CTT GAT GAT TCG CTC & 100 \\
& C & & \\
Il12a & CCA CCC TTG CCC TCC TAA & GCC GTC TTC ACC ATG TCA & 121 \\
& A & TCT & \\
Il2 & CGG CAT GTT CTG GAT TTG & TCA TCA TCG AAT TGG CAC & 134 \\
& AC & TC & \\
Ifng & CTG ATC CTT TGG ACC CTC & ACA GCC ATG AGG AAG AGC & 121 \\
& TG & TG & \\
Il10 & GCC TTA TCG GAA ATG ATC & TGA GGG TCT TCA GCT TCT & 115 \\
& CA & CAC & \\
Gzmb & GAC CCA GCA AGT CAT CCC & CCA GCC ACA TAG CAC ACA & 186 \\
& TA & TC & \\
Prf1 & CGG TGT CGT GTG GAA CAA & TCA TCA TCC CAG CCG TAG & 126 \\
& TA & TC & \\
Foxp3 & CTG CCT TGG TAC ATT CGT & CCA GAT GTT GTG GGT GAG & 101 \\
& GA & TG & \\
\hline
\end{tabular}

Ifng, interferon $\gamma$; Gzmb, granzyme b; Prf1, perforin; Foxp3, forkhead box P3 